\title{
Hybrid Modeling Method for Soft Sensing of Key Process Parameters in Chemical Industry
}

\author{
Hong Zhou, ${ }^{1,2^{*}}$ Kun-Ming Yu, ${ }^{3}$ and Huan-Po $\mathrm{Hsu}^{2}$ \\ ${ }^{1}$ Faculty of Computer and Software Engineering, Huaiyin Institute of Technology, \\ 1 Meicheng Rd, Huaian 223003, Jiangsu, P.R. China \\ ${ }^{2}$ Ph.D. Program in Engineering Science, College of Engineering, Chung Hua University, \\ 707, Sec.2, WuFu Rd., Hsinchu 30012, Taiwan \\ ${ }^{3}$ Department of Computer Science and Information Engineering, Chung Hua University, \\ 707, Sec.2, WuFu Rd., Hsinchu 30012, Taiwan
}

(Received November 25, 2020; accepted June 22, 2021)

Keywords: soft sensing, chemical industry, key process parameter prediction, LightGBM

Soft sensing technology is an effective way to solve the problem that important quality indicators of processing industries cannot be detected online, especially in the chemical industry. Owing to the complex working conditions, strong nonlinearity, strong coupling, and timevarying characteristics of chemical production processes, how to establish a soft sensing model with good prediction performance has become a valuable research topic. A soft sensing model based on a single-model method cannot guarantee global prediction accuracy, and the model stability is poor. A hybrid modeling method can integrate different modeling methods to describe the process characteristics of an object more comprehensively, so as to significantly improve the prediction accuracy and stability of the soft sensing model. In this paper, the key process parameter (solid-liquid ratio) in the evaporation salt (ES)-making process is taken as an example to carry out the following research. Firstly, aiming at the problems of production data obtained from the chemical industry, such as missing values, data inconsistency, high dimensions, high correlation, and time-series characteristics of features, an effective feature extraction method is proposed. On this basis, two data-driven models, the deep neural network (DNN) model for non-temporal regression prediction and the long short-term memory neural network (LSTM) model for temporal regression prediction, are established, and the regression performance of these two soft sensing models is evaluated. Secondly, another feature selection method based on prior domain knowledge, expert experience, and data mining is proposed. On this basis, a hybrid soft sensing model, the LightGBM model, is constructed for key process parameter prediction under different feature inputs, and the regression performance is evaluated. Simulation results demonstrate that introducing domain knowledge and expert experience to the modeling can enhance the interpretability of models, simplify the molding process, and further improve model performance.

*Corresponding author: e-mail: homezhou@pdlab.csie.chu.edu.tw https://doi.org/10.18494/SAM.2021.3436 


\section{Introduction}

To attain good quality control, many manufacturers carry out real-time and accurate detection of product quality or important process variables. The important process variables, also called key quality indicators, are closely related to product quality and have a great impact on production process quality control and optimization. However, in some typical processing industries, such as the chemical industry, it is difficult or dangerous to measure key quality indicators in real time. Soft sensing was proposed by Brosilow and Tong as a methodology to resolve the problem of difficult measurement. ${ }^{(1)}$

The evaporation salt (ES)-making process in salt chemical production is taken as the processing industry scenario. Evaporation process data (clearly marked data) from an actual salt chemical enterprise are selected as the study data for the chemical industry, and the solid-liquid ratio, a crucial progress quality indicator, is chosen to be monitored and predicted as the softsensing object.

On the basis of the process data and prior domain-specific knowledge, various judicious dataprocessing methods are designed to resolve problems such as high dimensions, mixed data types, missing values, duplicate values, and abnormal values. In addition, three different core production parameter (solid-liquid ratio) prediction models for the chemical industry are established in which input-related production parameters are inputted at a certain time and the solid-liquid ratio at the current time is outputted. In this way, the manufacturer can make an advance judgement on whether the solid-liquid ratio is within the range of golden threshold values, so as to ensure process stability, avoid the loss caused by a process quality decline or fluctuation, improve the product quality and output, and realize energy conservation and environmental protection.

As reported in this paper, we aim to design universal modeling methodologies for the chemical industry based on data and knowledge data; to propose useful methods for common and classical data quality issues, such as missing values, the high correlations and dimensions of features, and the temporality of features; and to realize effective prediction models for the regression analysis of key quality variables.

\section{Related Work}

Generally, the modeling method for soft sensing in industrial processes can be roughly classified into three categories: mechanism modeling, data-driven modeling, and hybrid modeling. With the progress of intelligent sensing technology and data mining, more online or offline applications of intelligent soft sensing have been developed, which utilize easily measured process variables to estimate difficult-to-measure variables. In principle, the development of soft sensors can be regarded as a regression problem, so various supervised machine learning algorithms have been applied.

Li et al. designed a genetic neural fuzzy system and used the genetic algorithm and backpropagation algorithm to train the model to improve system accuracy and accurately predict the weight of injection molded parts. ${ }^{(2)}$ Wang et al. applied a novel soft sensing method based on 
partial least-squares regression to perform the real-time measurement of product quality in a refining process. ${ }^{(3)}$ Pani et al. developed a backpropagation neural network for a rotary cement kiln for the soft sensing of clinker quality parameters. ${ }^{(4)}$ Liu et al. proposed a hybrid method called the least-squares support vector machines with ant colony-immune clone particle swarm optimization for soft sensing of the key quality variable MI in propylene polymerization. ${ }^{(5)}$ Aye et al. combined linear regression with a classification module, aiming to solve the problem that the aging of a telescopic probe affects the detection results of semiconductor quality. ${ }^{(6)}$ Nawaz et al. proposed an artificial neural network (ANN) and four hybrid neural networks (PCA-Kalman NN, PCA NN, Kalman NN, and non-NN) for data-driven soft sensing in a sidestream anammox process. ${ }^{(7)} \mathrm{Zhu}$ et al. summarized the application of deep learning in datadriven soft sensing modeling methods. ${ }^{(8)}$

In summary, conventional regression methodologies, optimization algorithms, and new intelligent techniques can be effectively applied in data-driven soft sensing. However, there has been insufficient research and attention paid to hybrid modeling methods.

\section{Methodology}

Manufacturing processes in the chemical industry are complex, uncertain, and nonlinear, which make it more difficult to build an accurate mathematical model. In this study, three universal modeling methodologies for processing industries to perform key quality variable prediction based on data (Methodologies 1 and 2) and based on knowledge data (Methodology 3) are proposed as shown in Fig. 1. Different prediction models are used in the methodologies: the deep neural network (DNN) for Methodology 1, long short-term memory (LSTM) for Methodology 2, and light gradient boosting machine (LGBM) for Methodology 3. Furthermore,

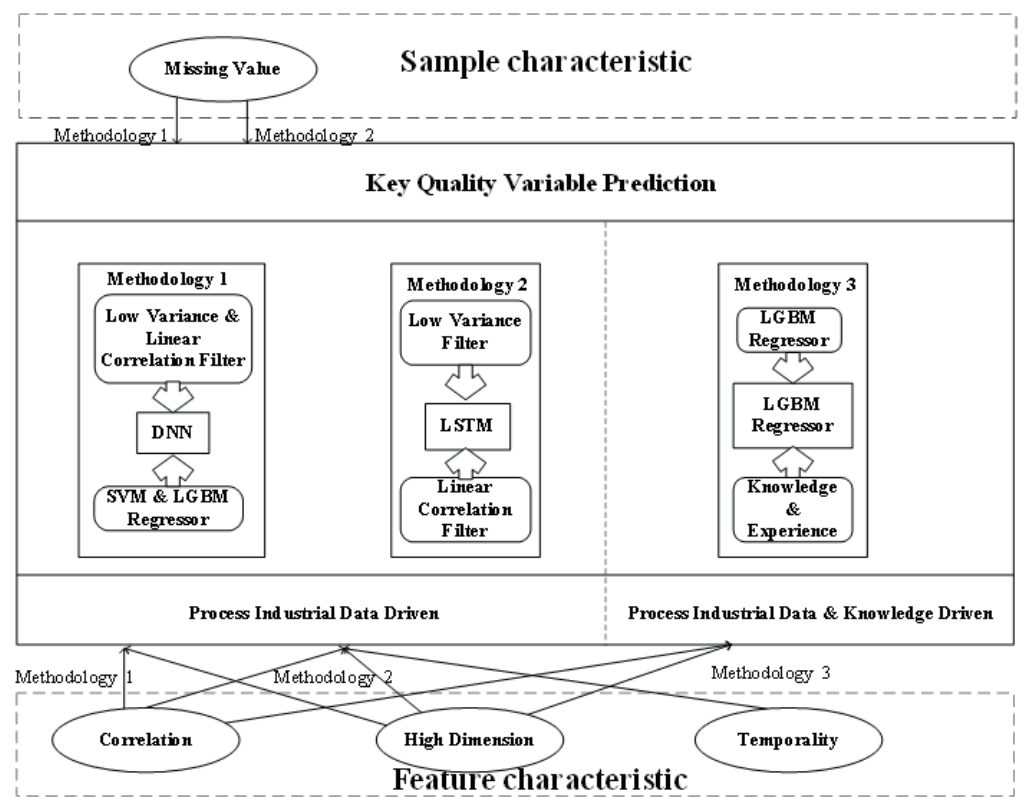

Fig. 1. Three methodologies for key quality variable prediction. 
effective methods and models are proposed to solve common and classical feature issues, such as high correlations and dimensions, and time sequences. In Methodology 1, low-variance and linear correlation filters and the SVM-LGBM regressor are adopted, whereas in Methodology 2, only filters are applied. However, Methodology 2 resolves the time sequence issue through the LSTM model. In Methodology 3, the LGBM regressor combined with knowledge and experience is utilized for features with a high correlation and dimension.

\subsection{Business analysis of production process}

For the main salt-making process of vacuum ES making in the modern chemical industry, the solid-liquid ratio is a core process index that has a great impact on the quality of the salt produced. The prior knowledge related to the manufacturing process of sodium nitrate identifies five production parameters as the vital quality indicators for the solid-liquid ratio, which are listed in Table 1. In addition, the collection of the following six production parameters in the same production equipment to predict the solid-liquid ratio is recommended by an expert from manufacturing: the discharge flow of salt slurry from the salt leg; the outlet temperature of the feed liquid; the inlet temperature of the feed liquid; the liquid level; the actual value of flow control for salt leg washing; the actual value of brine flow control.

\subsection{Data analysis of production process}

The ES dataset adopted in this study is obtained from a real salt chemical enterprise. The ES dataset is collected from the core equipment contained in the sodium nitrate production process in the salt production line by vacuum evaporation.

This dataset has 43201 records, indexed from 0 to 43200. Only 10 records of this dataset contain a null value, which will not be a problem because of the small number compared with the number of records (43201). This dataset has 1936 features (discrete and continuous features coexist). Each feature is named by the enterprise using a letter and symbol, which are abstract

Table 1

Description of production parameters affecting solid-liquid ratio in domain knowledge.

\begin{tabular}{lccl}
\hline No. & Name & Value scope & \multicolumn{1}{c}{ Description } \\
\hline 1 & Steam pressure & $>0.45$ & Steam pressure is relatively high for better production. \\
\hline 2 & Steam temperature & 260 & $\begin{array}{l}\text { Steam temperature is usually related to steam pressure and should not be } \\
\text { too high, normally } 260^{\circ} \mathrm{C} .\end{array}$ \\
\hline $\begin{array}{c}\text { Current value of } \\
\text { circulating pump }\end{array}$ & $470-550$ & $\begin{array}{l}\text { If the current value of the circulating pump continues to rise, the solid- } \\
\text { liquid ratio should be high and the opening of the salt leg valve must be } \\
\text { increased; if it continues to decrease, the solid-liquid ratio should be low } \\
\text { and the opening of the salt leg valve must be reduced; if the current rises } \\
\text { suddenly, the pipeline should be blocked. }\end{array}$ \\
\hline $\begin{array}{c}\text { Opening of salt leg } \\
\text { valve }\end{array}$ & $0-100$ & $\begin{array}{l}\text { When the valve is fully open, the salt leg volume should be } 60 \mathrm{~m}^{2}, \\
\text { and if it is } 40 \mathrm{~m}^{2}, \text { it means that the pipeline is obstructed. }\end{array}$ \\
\hline 5 & $\begin{array}{c}\text { Salt discharge of } \\
\text { salt leg }\end{array}$ & $0-90$ & $\begin{array}{l}\text { When the salt is fully discharged, the effect of changing the solid-liquid } \\
\text { ratio can be seen in about } 10 \text { min. When the salt discharge is small, it may } \\
\text { take about } 30 \text { min. }\end{array}$ \\
\hline
\end{tabular}


and difficult to understand. However, the full name and the practical role of each feature in production can be understood through the data dictionary provided by the enterprise.

In summary, the ES dataset is a typical dataset of a processing industry that contains the following problems that need to resolved: (1) Inconsistency of data format: There are 718 discrete columns whose value is a character type, including a time column and a column indicating the state of the switch. (2) High number of dimensions: The number of features in the original dataset is as high as 1934. (3) Time-varying: For the chemical processing industry, the time variation of its parameters (such as pressure, temperature, flow, etc.) in a time series cannot be ignored in the data analysis.

\subsection{Design of regression model based on nontemporal data}

Machine learning, data mining, and other technologies are used to build a regression model based on nontemporal manufacturing process data, and the modeling process is shown in Fig. 2.

(1) Data preprocessing

A. Discrete column digitization

The discrete column is used to represent the switch in the dataset using the character type, whose value is "OFF" or "ON". After digitization, "OFF" is set to 0 and "ON" is set to 1 .

B. Feature selection using low-variance filter

The variance reflects the difference between most of the values and their average values. If the difference is large, the variance will be large, and if the difference is small, the variance will be small. Low-variance filtering is performed directly on the raw data for feature

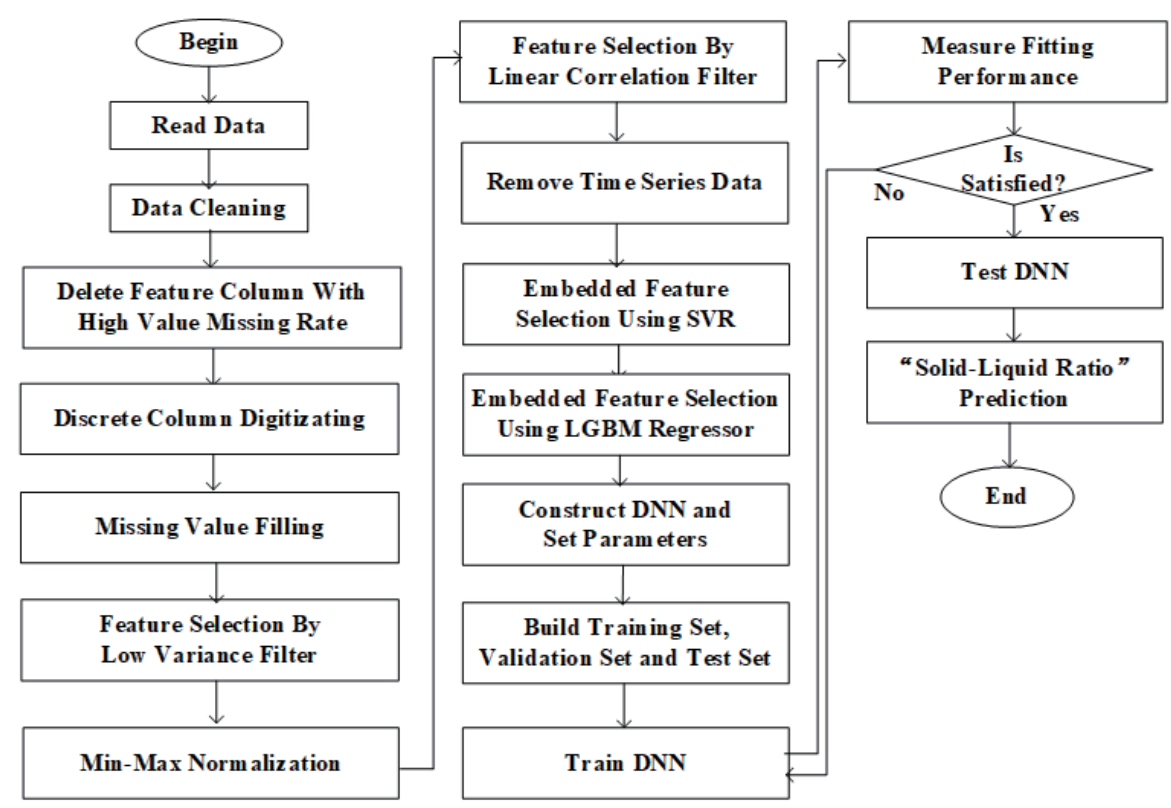

Fig. 2. Modeling process for key quality variable based on nontemporal process data. 
filtering according to a threshold. If the feature's variance is less than the threshold, this feature will be identified as having no effect on sample discrimination, and it is discarded.

C. Normalization using min-max method

D. Feature selection by linear correlation filter

The linear correlation between features is calculated, features with extremely strong correlation are found, then, according to the association path, features with the largest variance are retained.

E. Remove time-series data

(2) Missing value filling

Although the proportion of missing values in this dataset is small, missing value interpolation is still performed. Discrete features are filled with the mode value, and continuous features are filled with the mean value. The mode value refers to a value reflecting the centralized trend in the statistical distribution, which is likewise the most frequently occurring value in a dataset.

(3) Embedded feature selection using support vector regressor

The basic idea of the support vector regression (SVR) algorithm is to find a regression plane using Eq. (1), so that all the data in a set are closest to the plane, ${ }^{(9)}$

$$
f(x)=\sum_{i=1}^{l}\left(a_{i}-a_{i}^{*}\right) k\left(x_{i}, x\right)+\beta,
$$

where $x$ is the input variable; $a_{i}$ and $a_{i}{ }^{*}$ are Lagrange multipliers $\left(a_{i}, a_{i}{ }^{*} \geq 0\right) ; \beta$ is bias; $\varphi$ is the mapping function; $k\left(x_{i}, x\right)$ is the kernel function (which is a linear function in this section).

(4) Embedded feature selection using LightGBM regressor

The gradient boosting decision tree (GBDT) using the gradient-based one-side sampling (GOSS) algorithm and exclusive feature bundling (EFB) algorithm is called LightGBM. ${ }^{(9)}$ Feature selection by LightGBM adopts the gain of each feature when the tree model splits nodes to evaluate the importance of features. Other feature selection algorithms take the number of feature splittings as the evaluation method.

A. The core function of GOSS is to optimize sampling optimization samples of the training set.

a. Samples with a larger gradient are retained.

b. Samples with a small gradient are randomly sampled.

c. When calculating the gain, the weight coefficient is added to the samples with a small gradient.

B. The EFB algorithm is used for feature extraction, binding mutually exclusive features (one feature value is zero, one feature value is not zero), and reducing the feature dimension.

a. Input: feature $\mathrm{F}$, maximum number of conflicts $\mathrm{K}$, graph $\mathrm{G}$.

b. Output: feature bundles.

c. A graph with weighted edges is constructed, which corresponds to the total conflict between features. The features are sorted in descending order by their degree in the graph. 
Each feature in the ordered table is examined and assigned to an existing bundling with minor conflicts, or a new bundle is created.

(5) Regression analysis by DNN

A fully connected feedforward DNN is used for regression analysis.

(6) Measure fitting performance

The root mean square error (RMSE) is adopted to measure the fitting performance of the regressor, which indicates the error between the predicted variable value and the actual value. The mathematical definition of RMSE is

$$
R M S E=\sqrt{\frac{\sum_{i=1}^{n}\left(X_{o b S, i}-X_{p r e, i}\right)^{2}}{n} .}
$$

\subsection{Design of regression model based on time-series data}

By focusing on time-series data, the time-varying characteristics of the production process are introduced to better mine the potential production rule. A regression model based on manufacturing process data is designed as shown in Fig. 3.

The modeling process shown in Fig. 3 is very similar to that in Fig. 2, except that time-series data are added to the dataset, and the prediction model is an LSTM network. The basic idea of the LSTM is to introduce a gate control device to deal with the problems of memory/forgetting, input degree, and output degree.

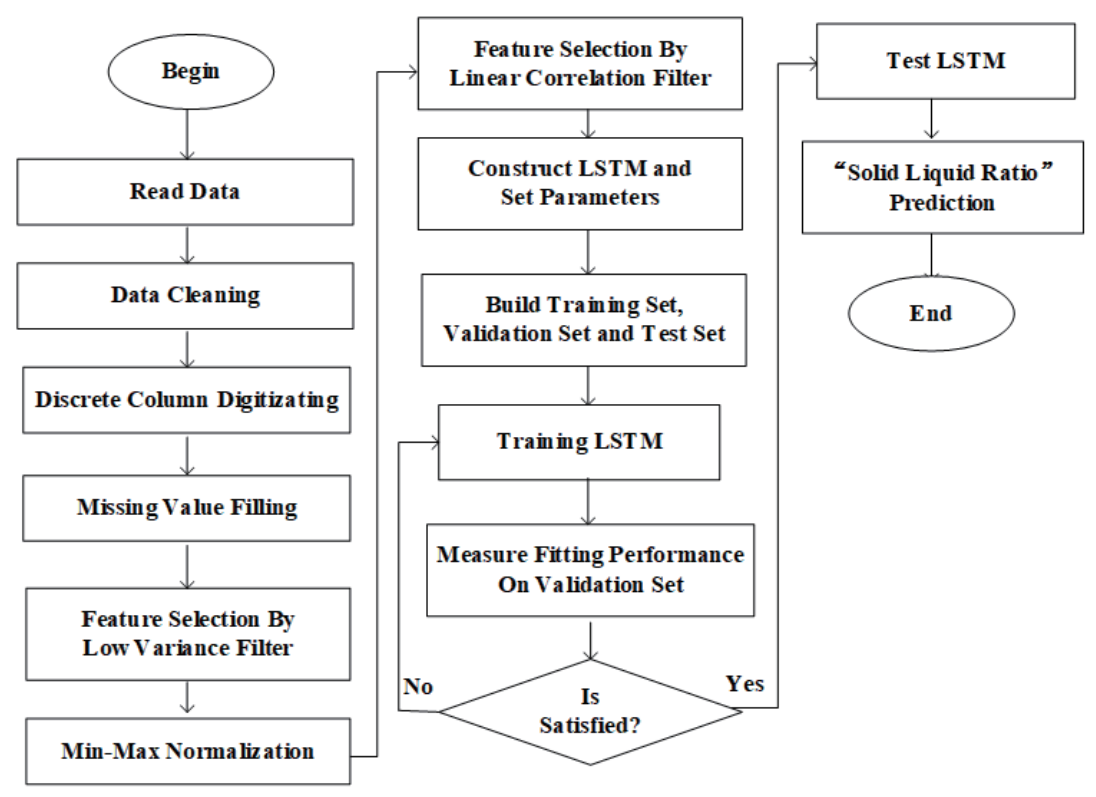

Fig. 3. Modeling process for key quality variable based on time-series data. 


\subsection{Design of regression model based on knowledge and data}

Prior knowledge and expert experience are introduced to the model shown in Fig. 4, which is particularly meaningful for most engineering applications, especially process engineering applications. LightGBM, an upgraded framework based on the GBDT algorithm, is applied to realize feature selection and regression analysis at the same time. ${ }^{(10,11)}$ Feature selection by LightGBM is based on a tree model such as extreme gradient boosting (XGBoost). It is composed of two parts: a decision tree algorithm and a gradient boosting algorithm.

"Light" mainly refers to three aspects: fewer samples, fewer features, and less memory, which is realized through GOSS, EFB, and a histogram. The regression process based on LightGBM is described as follows.

(1) Input data to the first tree and predict.

(2) Calculate the residual error between the learning result of the previous tree and the final regression target and use it as the learning target of the second tree. The attenuation should be multiplied to prevent overfitting in this step.

(3) Repeat the above learning process until the end.

(4) Calculate the final predicted value as the sum of the predicted values of each tree.

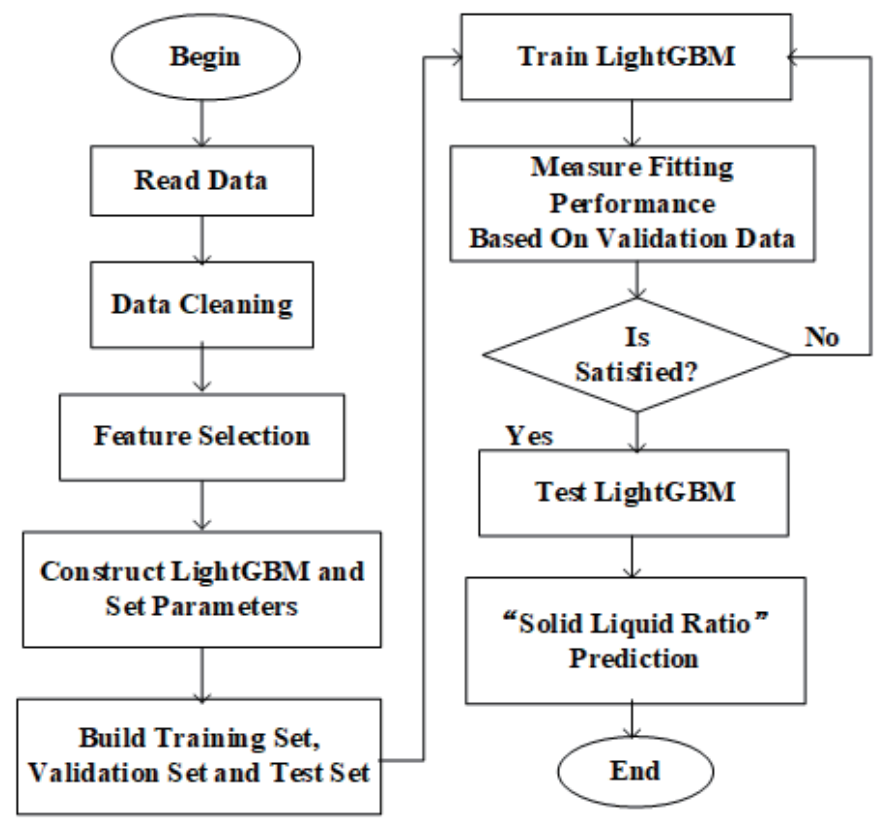

Fig. 4. Modeling process for key quality variable based on knowledge, experience, and data. 


\section{Simulation and Evaluation}

\subsection{Regression analysis based on nontemporal data}

The simulation for the regression analysis based on nontemporal manufacturing process data shown in Fig. 2 is illustrated as follows.

(1) Data preprocessing

A. Discrete column digitization: Perform digitization on 717 columns, which are all discrete columns, except for the time column.

B. Feature selection using low-variance filter: Do low-variance filtering directly on the raw data without normalization, setting the variance threshold to be 0.0001 . After filtering, 571 features are retained and 1362 features are discarded.

C. Normalization using min-max method.

D. Feature selection by linear correlation filter: Find 429 features with extremely strong correlation (linear correlation greater than 0.8 or less than -0.8 ) and, according to the association path, discard 312 features.

E. Remove time-series data.

(2) Filling in missing values

(3) Embedded feature selection using support vector regressor

The weight of features calculated using the support vector regressor is used for feature selection. The features whose weight values are in the top $25 \%$ of all feature weight values are selected as important features. After operation, 194 important features are selected.

(4) Embedded feature selection using LightGBM regressor

The weight of features calculated using the LightGBM regressor is used for further feature selection and important features are selected. The quantile of the feature weight is calculated, and the quantile whose weight score is greater than or equal to 10 is selected as an important feature. After further selection, 84 important features are selected.

(5) Build dataset

The dataset is randomly divided in the ratio of 70:30 for training and testing, respectively, and fivefold cross-validation is performed.

(6) Regression analysis by DNNs

A fully connected feedforward DNN model is built. There are 64 nodes in the first layer, 32 nodes in the second layer, 16 nodes in the third layer, and one node in the fourth layer. The first three layers use the ReLU activation function. The dataset is input to this model for training, validation, and testing. Then the experimental results are analyzed, the parameters of the model are adjusted, and then training, validation, and testing are carried out again. 
The experimental results discussed in this section are based on the test dataset, in which the number of samples is 12963 and the feature dimension is 85 . The RMSE of the prediction model for the solid-liquid ratio is 0.1 , which means that the model has a perfect prediction performance. Furthermore, the feature reduction in this section performed using the combined feature selection model also works well, which reduces the feature dimension from 1936 to 84 . The number of feature dimensions of the ES dataset is thus decreased by $95.6 \%$.

\subsection{Regression analysis based on time-series data}

Since many of the simulation steps shown in Fig. 3 are similar to those in Fig. 2, in this section, only the construction of the LSTM model by "Sequential" in Keras (the Python deep learning API), which is a way to organize the network layer sequentially, is described. The first layer is the embedding layer for the input; the second layer is the LSTM layer with a dropout rate of 0.2 between the first layer and the second layer; the third layer is the dense layer with a dropout rate of 0.2 between the second layer and the third layer, which is a fully connected layer that uses the ReLU activation function.

All results discussed in this section are based on the test dataset, in which the number of samples is 12963 and the feature dimension is 86 (85 selected important features plus one temporal feature). The RMSE of the prediction model for the solid-liquid ratio is 0.38 , which means that this model has a good prediction performance.

\subsection{Regression analysis based on knowledge and data}

The simulation for the regression analysis based on knowledge and data shown in Fig. 4 is described as follows.

(1) Feature selection based on prior knowledge

According to the prior knowledge, the vital features listed in Table 2 related to the selected production equipment and line are found from the original dataset. Only five columns (all continuous data) are selected on the basis of prior knowledge.

(2) Feature selection based on expert experience

According to expert experience, in addition to the features selected on the basis of prior knowledge, there are also some features related to the current equipment in production that must be selected. Only the five features (all continuous data) listed in Table 3 are selected on the basis of prior knowledge.

(3) Feature selection based on LightGBM

Construct the LightGBM: Evaluate the importance of features F0-F9 selected on the basis of knowledge and expert experience. List the output features in descending order according to the importance score, and select features based on the importance score. Four features (F3, F6, F5, F0) with the highest importance scores are selected. 
(4) Feature selection based on LightGBM combined with prior knowledge

Check the features selected in Step 3 to establish whether the vital features (F0-F4) are all included. If they are all included, do not make any changes; if not, add the missed features. As a result, seven features (F3, F6, F5, F0, F1, F2, F4) are selected.

Table 2

Description of vital features selected on the basis of prior knowledge.

\begin{tabular}{ll}
\hline No. & Description \\
\hline F0 & Flow control of salt slurry discharge from salt leg \\
F1 & Flow control of actual value of salt leg salt slurry discharge \\
F2 & Preset value of flow control for salt leg salt slurry discharge \\
F3 & Steam pressure \\
F4 & Discharge flow of salt slurry from salt leg \\
\hline
\end{tabular}

Table 3

Description of vital features selected on the basis of expert experience.

\begin{tabular}{ll}
\hline No. & Description \\
\hline F5 & Outlet temperature of feed liquid \\
F6 & Inlet temperature of feed liquid \\
F7 & Liquid level \\
F8 & Actual value of flow control for salt leg washing \\
F9 & Actual value of brine flow control \\
\hline
\end{tabular}

Table 4

Comparison of prediction performances based on different features.

\begin{tabular}{lcl}
\hline RMSE & Feature dimension & \multicolumn{1}{c}{ Feature description } \\
\hline 2.53 & 5 & Selected on the basis of prior knowledge: F0-F4 \\
2.10 & 10 & Selected on the basis of knowledge and expert experience: F0-F9 \\
2.65 & 4 & Selected on the basis of machine learning: F3, F6, F5, F0 \\
0.08 & 7 & Selected by machine learning and prior knowledge: F3, F6, F5, F0, F1, F2, F4 \\
\hline
\end{tabular}

Construct the LightGBM model with classical or default parameter values. Input the dataset into this model for training, validation, and testing. All results discussed in this section are based on test datasets in which the number of samples is the same but the feature dimension and content are different. The average RMSE values are listed in Table 4. In the experiments, the same regressor is applied, the same number of samples is input, and only the features are different. However, there is a huge performance gap between the best performance (RMSE of 0.08 ) and the worst performance (RMSE of 2.65); the RMSE is decreased by $97 \%$. This dramatic performance difference originates from the different feature selection methods. The features used in the experiment with the best performance are selected on the basis of knowledge data mining. The features used in the experiment with the worst performance are selected on the basis of machine learning. The experiments demonstrate that the physical meanings of the features are important for feature selection and modeling and that domain-specific knowledge is helpful in feature selection and modeling. 


\section{Conclusions}

Modeling is the cornerstone of engineering data analysis. By taking the data modeling of the salt-making process as an example, an in-depth study is carried out on the background for key quality variable prediction in the chemical industry.

We first design various feature selection methods for general industrial data. First, the feature selection methods of the low-variance filter, high-correlation filter, embedded SVR regressor, and LightGBM regressor are applied synthetically or separately. In experiments in which these methods are applied synthetically or two filters are applied synthetically, there is no significant difference in feature dimension reduction, with the feature dimension reduced from 1936 to 84 and 86, respectively. Second, knowledge is introduced into the LightGBM regressor to perform feature selection, and the feature dimension is reduced from 1936 to 7. It is verified by experiment that all these methodologies for feature selection can effectively resolve the problems of high correlation and high dimension at the same time.

In addition, we design various quality variable prediction models for the chemical industry. The first is based on a DNN using nontemporal data, the second is based on LSTM with timeseries data introduced, and the third is the LightGBM regressor with prior domain knowledge and expert experience introduced for use as a gray-box model. The experimental results show that the prediction RMSE of non-time-series regression is 0.1, the RMSE of regression considering time variation is 0.38 , and the RMSE of regression after introducing knowledge into the LightGBM regressor is 0.08 .

In summary, compared with a model based on data, introducing knowledge can have a positive effect on feature dimension reduction and regression performance at the same time. In other words, introducing domain knowledge into modeling can enhance the interpretability of models, simplify the modeling process, and improve the model performance. In future research, a core issue will be how to better integrate the data-driven method for perception and the knowledge-driven method for cognition in a hybrid model to enhance the robustness and universality of the model

\section{Acknowledgments}

This research was supported in part by the Ministry of Science and Technology of R.O.C. under contract MOST 105-2221-E-216-015-MY2.

\section{References}

1 C. Brosilow and M. Tong: Aiche J. 24 (2010) 230. https://doi.org/10.1039/b918972f

2 E. Li, L. Jia, and J. Yu: Comput. Chem. Eng. 26 (2002) 1253. https://doi.org/10.1016/S0098-1354(02)00092-3

3 D. Wang, J. Liu, and R. Srinivasan: IEEE Trans. Ind. Inf. 6 (2010) 11. https://doi.org/10.1109/TII.2009.2025124

4 A. K. Pani, V. Vadlamudi, R. J. Bhargavi, and H. K. Mohanta: Proc. 2011 IEEE Int. Conf. Process Automation, Control and Computing (IEEE, 2011) 1-6.

5 Y. Liu, Z. Gao, and J. Chen: Chem. Eng. Sci. 102 (2013) 602. https://doi.org/10.1016/j.ces.2013.07.002

6 T. T. Aye, F. Yang, L. Wang, G. K. K. Lee, and M. C. Nguyen: Proc. 2015 IEEE 10th Conf. Industrial Electronics and Applications (IEEE, 2015) 345-350. 
7 A. Nawaz, A. S. Arora, C. M. Yun, H. C. Cho and M. Lee: Ind. Eng. Chem. Res. 58 (2019) 9552. https://doi. org/10.1021/acs.iecr.9b00722

8 X. Zhu, K. U. Rehman, B. Wang, and M. Shahzad: Sens. J. 20 (2020) 1771. https://doi.org/10.3390/s20061771

9 R. G. Brereton and G. R. Lloyd: Anal. J. 135 (2010) 230. https://doi.org/10.1039/B918972F

10 G. L. Ke, Q. Meng, T. Finley, T. F. Wang, W. Chen, W. D. Ma, Q. W. Ye, and T. Y. Liu: Proc. 31st Int. Conf. Neural Information Processing Systems (NIPS, 2017) 3146-3154.

11 T. Q. Chen and C. Guestrin: Proc. 22nd ACM SIGKDD Int. Conf. Knowledge Discovery and Data MiningKDD '16 (KDD, 2016) 785-794.

\section{About the Authors}

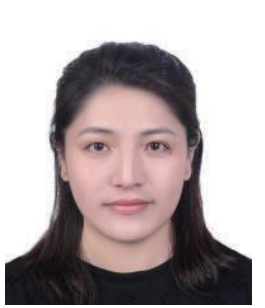

Hong Zhou received her B.S. degree in computer science and technology from Huaiyin Institute of Technology, Huaian, Jiangsu, China in 2004, her M.S. degree in internet computing from the University of Abertay Dundee, Dundee, UK, in 2008, and her Ph.D. degree in engineering science from Chung Hua University, Hsinchu, Taiwan, in 2020. Since 2004, she has been a teacher in the Faculty of Computer and Software Engineering, Huaiyin Institute of Technology. Currently, she is an associate professor in the Faculty of Computer and Software Engineering, Huaiyin Institute of Technology. Her research interests include machine learning, data mining, big data, and artificial intelligence.

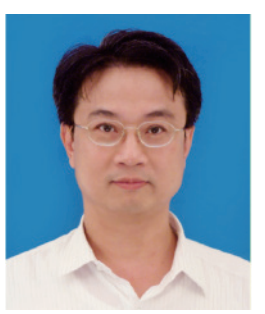

Kun-Ming Yu received his B.S. degree in chemical engineering from National Taiwan University in 1981, and his M.S. and Ph.D. degrees in computer science from the University of Texas at Dallas in 1988 and 1991, respectively. From 2005 to 2017, he was the dean of the College of Computer Science and Informatics, Chung Hua University. He is currently a vice president of Chung Hua University. His research interests include artificial intelligence, big data, IoT, high-performance computing, ad hoc networks, and computer algorithms.

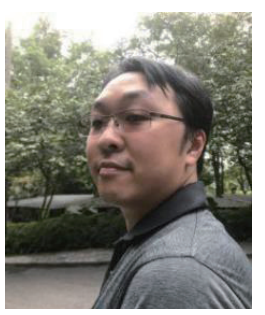

Huan-Po Hsu received his B.S. degree in mechanical engineering from Chinese Culture University in 2013 and his M.S. degree in computer science and information engineering from Chung Hua University in 2015. He is currently pursuing his Ph.D. degree in engineering science from Chung Hua University. He is the chairman of SmartPearls Co., Ltd. His research interests include IoT and intelligent evacuation guiding technology. 